\title{
Abnormal expression of leiomyoma cytoskeletal proteins involved in cell migration
}

\author{
BLENDI URA $^{1}$, FEDERICA SCRIMIN ${ }^{1}$, GIORGIO ARRIGONI ${ }^{3,4}$, EMMANOUIL ATHANASAKIS ${ }^{1}$, \\ MICHELANGELO ALOISIO ${ }^{1}$, LORENZO MONASTA ${ }^{1}$ and GIUSEPPE RICCI ${ }^{1,2}$ \\ ${ }^{1}$ Institute for Maternal and Child Health - IRCCS 'Burlo Garofolo', Trieste; ${ }^{2}$ Department of Medical, \\ Surgery and Health Sciences, University of Trieste, Trieste; ${ }^{3}$ Department of Biomedical Sciences, \\ University of Padova, Padova; ${ }^{4}$ Proteomics Center of Padova University, Padova, Italy
}

Received October 2, 2015; Accepted November 5, 2015

DOI: $10.3892 / o r .2016 .4688$

\begin{abstract}
Uterine leiomyomas are monoclonal tumors. Several factors are involved in the neoplastic transformation of the myometrium. In our study we focused on dysregulated cytoskeletal proteins in the leiomyoma as compared to the myometrium. Paired tissue samples of ten leiomyomas and adjacent myometria were obtained and analyzed by two-dimensional gel electrophoresis (2-DE). Mass spectrometry was used for protein identification, and western blotting for 2-DE data validation. The values of ten cytoskeletal proteins were found to be significantly different: eight proteins were upregulated in the leiomyoma and two proteins were downregulated. Three of the upregulated proteins (myosin regulatory light polypeptide 9, four and a half LIM domains protein 1 and LIM and SH3 domain protein 1) are involved in cell migration, while downregulated protein transgelin is involved in replicative senescence. Myosin regulatory light polypeptide 9 (MYL9) was further validated by western blotting because it is considered to be a cell migration marker in several cancers and could play a key role in leiomyoma development. Our data demonstrate significant alterations in the expression of cytoskeletal proteins involved in leiomyoma growth. A better understanding of the involvement of cytoskeletal proteins in leiomyoma pathogenesis may contribute to the identification of new therapeutic targets and the development of new pharmacological approaches.
\end{abstract}

\section{Introduction}

Uterine leiomyomas are monoclonal tumors in which interactions of sex steroids, somatic mutations and growth factors

Correspondence to: Dr Blendi Ura, Institute for Maternal and Child Health - IRCCS 'Burlo Garofolo', I-34137 Trieste, Italy

E-mail: blendi.ura2006@libero.it

Key words: cytoskeleton, leiomyoma, proteomics, myometrium, two-dimensional gel electrophoresis are involved in the neoplastic transformation of the myometrium (1). Several studies suggest that ovarian hormones and genetic factors may have a role in leiomyoma growth (2). The extracellular matrix (ECM) of the leiomyoma is constituted predominantly of collagens, proteoglycans and matrix glycoproteins, and their overproduction is directly involved in leiomyoma volume expansion (3). The development of the fibroid is due to the hyper-production of collagen and to the lack of degradation (4).

The leiomyoma is characterized by cytoskeletal alterations influencing contractility, cell migration and cell proliferation (5). TNF- $\alpha$ upregulates MMP-2 expression and stimulates cell migration through the extracellular signal-regulated kinase (ERK) pathway in the leiomyoma (6). Integrin plays a key role in cytoskeletal remodeling towards activation of RHOA (transforming protein RhoA), which takes part in cell signaling leading to cell contractility, ECM stiffening and tumor growth (7).

RHOA is essential in the regulation of the signal transduction pathway, and is involved in a microtubule-dependent signal required for the myosin contractile ring formation during cell cycle cytokinesis (8). Cell migration requires cytoskeletal rearrangement and RHOA is crucial in the regulation of this mechanism: by activating MYL9 (myosin regulatory light polypeptide 9), it increases the metastatic potential in cancer cells (9).

Several factors can induce alterations in the mechanotransduction signal from ECM via the transmembrane receptor to the interior of the cell (10). This signal causes the reorganization of the actin cytoskeleton, mediated by RHOA, inducing cell proliferation (11).

In this study, we present the data of the altered expression of several cytoskeletal proteins involved in cell migration in the tissue of the leiomyoma compared to the normal myometrium.

\section{Materials and methods}

Tissue samples were obtained from ten premenopausal patients who underwent hysterectomy for symptomatic uterine leiomyomas. The procedures complied with the Declaration of Helsinki and were approved by the Review Board of the 
Institute for Maternal and Child Health - IRCCS 'Burlo Garofolo’ (Trieste, Italy). All subjects signed a written informed consent.

The median age of patients was 46.5 with a minimum of 42 years and a maximum of 52 years.

Tissue samples. Two samples were collected from each patient: one from the central area of the leiomyoma and one from the unaffected myometrium. All the leiomyomas were confirmed histologically as benign ordinary leiomyomas. Samples were stored at $-80^{\circ} \mathrm{C}$ until proteomic analysis was performed.

Two-dimensional gel electrophoresis. Clean leiomyoma and myometrium (300 mg each) were homogenized in $1.2 \mathrm{ml}$ of dissolution TUC buffer [7 $\mathrm{M}$ urea, $2 \mathrm{M}$ thiourea, 4\% CHAPS, $40 \mathrm{mM}$ Tris, $65 \mathrm{mM}$ DTT and 0.24\% Bio-Lyte (3-10)] with a protease inhibitor mix (2 mM PMSF, $1 \mathrm{mM}$ benzamidine, $1 \mathrm{mM}$ EDTA, $1 \mathrm{mM} \mathrm{NaF}$ ) and a trace of bromophenol blue. The solutions were vortexed at maximum speed several times and kept at room temperature for $1 \mathrm{~h}$ and centrifuged at $10,000 \mathrm{x} \mathrm{g}$ at $4^{\circ} \mathrm{C}$ for $30 \mathrm{~min}$. The protein content of the supernatant was determined using the Bradford assay. Eight hundred micrograms of proteins from each sample were used for the 2-DE analysis.

NL IPG Readystrips, 18-cm, pH 3-10 (Bio-Rad, Hercules, $\mathrm{CA}, \mathrm{USA}$ ) were rehydrated at $50 \mathrm{~V}$ for $12 \mathrm{~h}$ at $20^{\circ} \mathrm{C}$. Isoelectric focusing (IEF) was performed in a Protein IEF cell (Bio-Rad) set to $170.000 \mathrm{Vh}$. After IEF, the IPG strips were equilibrated by serial incubation (20 min) in an equilibration buffer (6 M urea, $2 \%$ SDS, 50 mM Tris-HCl pH 8.8, 30\% glycerol, and 1\% DTT) and in an equilibration buffer containing $4 \%$ iodoacetamide instead of DTT. Equilibrated IPG strips were transferred onto a $12 \%$ polyacrylamide gel for the second dimension. After the second dimension, gels were fixed and stained for $48 \mathrm{~h}$ with colloidal Coomassie Blue, and excess dye was removed with distilled water. On average, three experimental replicates were performed per sample. Molecular masses were determined by precision protein standard markers (Bio-Rad). 2-DE gels were scanned with a Molecular Imager PharosFX system (Bio-Rad) and the quantitative analysis of the spots was carried out using the ProteomeWeaver 4 program (Bio-Rad).

Quantification of spot levels. Spot normalization was automatically performed by the software and was based on a normalization algorithm intended for numerical analysis, which did not require any internal standard. For each gel an intensity factor was computed to ensure all normalization factors were as close to one as possible. The matching produced a list of super spots, which represent certain protein species present in the gel. For the correct matching, each super spot was manually controlled prior to normalization. For each matched pair of gels, the quotient between the pair-matched spot was calculated. The normalization factor was the median of these quotients (ProteomeWeaver 4 program; Bio-Rad).

Trypsin digestion and MS analysis. Spots from 2-DE were washed 4 times with $50 \mathrm{mM} \mathrm{NH}_{4} \mathrm{HCO}_{3}$ and acetonitrile (ACN; Sigma-Aldrich) alternatively and dried under vacuum in a SpeedVac system. Three microliters of $12.5 \mathrm{ng} / \mu 1$ sequencing grade modified trypsin (Promega, Madison, WI, USA) in
$50 \mathrm{mM} \mathrm{NH} \mathrm{HCO}_{3}$ was added to each gel spot and samples were digested overnight at $37^{\circ} \mathrm{C}$. Peptides were extracted with three changes of $50 \%$ ACN/0.1\% formic acid (FA; Fluka), dried under vacuum and stored at $-20^{\circ} \mathrm{C}$ until mass spectrometry (MS) analysis was performed.

Samples were dissolved in $10 \mu \mathrm{l}$ of $0.1 \%$ trifluoroacetic acid (TFA; Riedel-de Haën). One microliter of each sample was mixed with $1 \mu \mathrm{l}$ of matrix solution ( $\alpha$-cyano4-hydroxycinnamic acid; Fluka). Five $\mathrm{mg} / \mathrm{ml}$ in $70 \%$ $\mathrm{ACN} / 0.1 \% \mathrm{TFA}$ ) and $0.8 \mu \mathrm{l}$ of the resulting solution were spotted onto a stainless steel MALDI target plate for the MS analysis on the MALDI-TOF/TOF 4800 analyzer (AB Sciex, Framingham, MA, USA). The analysis was performed in a data dependent mode: a full MS scan was acquired from each sample, followed by MS/MS spectra of the ten most intense signals.

Few samples that could not be identified by MALDI-TOF/TOF analysis were further analyzed by LC-MS/MS on an LTQ-Orbitrap XL mass spectrometer (Thermo Fisher Scientific, Rockford, IL, USA), coupled with a nano-HPLC Ultimate 3000 (Dionex - Thermo Fisher Scientific). Samples were loaded onto an in-house pico-frit column packed with C18 material (ReproSil, $300 \AA$, $3 \mu \mathrm{m}$; Dr. Maisch HPLC GmbH) and separated using a 20-min linear gradient of ACN/0.1\% formic acid (from 3 to $40 \% \mathrm{ACN}$ ), at a flow rate of $250 \mathrm{nl} / \mathrm{min}$. Capillary voltage was set at $1.3-1.5 \mathrm{kV}$ and source temperature at $200^{\circ} \mathrm{C}$. The analysis was performed in a data dependent mode, and a full scan at 60,000 resolution on the Orbitrap was followed by MS/MS fragmentation scans on the ten most intense ions acquired with CID fragmentation in the linear trap.

MS and MS/MS spectra obtained from MALDI-TOF/TOF analysis were converted into MGF (Mascot generic format) files to be elaborated with Proteome Discoverer 1.4 (Thermo Fisher Scientific), while raw data files from the LTQ-Orbitrap XL mass spectrometer were directly analyzed with the software. Proteome Discoverer was interfaced to a Mascot search engine, version 2.2.4 (Matrix Science, London, UK).

The database used for protein identification was UniProt Human (version 20140709, 88993 sequences), while enzyme specificity was set to trypsin with 1 missed cleavage. The mass tolerance window was set to $10 \mathrm{ppm}$ for parent mass and to 0.6 Da for fragment ions for the files from LTQ-Orbitrap XL, while the tolerances were $50 \mathrm{ppm}$ (parent) and $0.3 \mathrm{Da}$ (fragment ions) for the MALDI-TOF/TOF data. Carbamidomethylation of cysteine residues was set to 'fixed modification' and methionine oxidation to 'variable modification'.

Proteome Discoverer calculates a false discovery rate (FDR) based on the parallel search against a randomized database. Proteins were considered as positive hits if at least two independent peptides were identified with medium (95\%) or high $(99 \%)$ confidence.

STRING 9.0 network analysis and biological functions. Possible connections among identified cytoskeletal proteins with significant variations as compared to normal myometrium versus leiomyoma were analyzed by a protein and gene network software. For each protein, related gene names were acquired in UniProtKB and used for network generation by the use of STRING 9.0 (http://www.string-db.org/). 


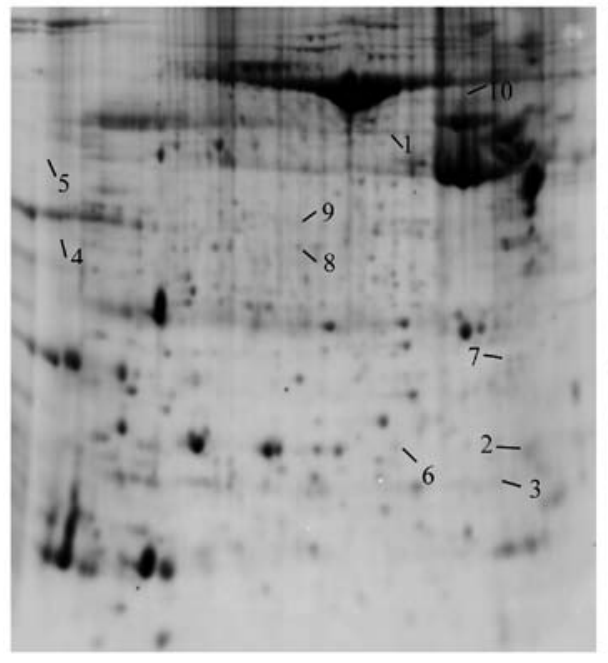

10

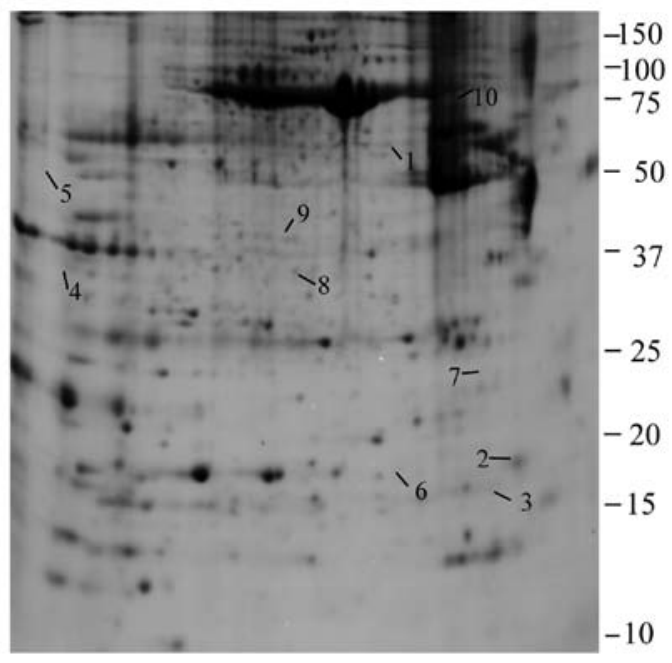

$10 \longleftarrow$

Myometrium

Figure 1. Two-dimensional electrophoresis map of normal myometrium and uterine leiomyoma proteome. Immobilized pH gradient 3-10 NL strip in the first dimension and a $12 \%$ polyacrylamide gel in the second dimension.

Differential proteins distributed in the biological function data were obtained from Gene Ontology (http://amigo.geneontology.org/rte).

Western blotting. Western blot analysis was performed as previously described (12). Protein extracts (30 $\mu \mathrm{g})$ used for 2 -DE were separated by $10 \%$ SDS-PAGE, and then transferred to a nitrocellulose membrane in a blotting chamber. The residual binding sites on the membrane were blocked by treatment with defatted dry milk proteins, and incubated overnight at $4^{\circ} \mathrm{C}$ with 1:1,000 diluted primary rabbit polyclonal antibody against myosin regulatory light polypeptide 9 (Sigma-Aldrich). After washing, membranes were incubated with an HRP-conjugated anti-rabbit IgG (Sigma-Aldrich) in a dilution of 1:3,000. At the end the protein expression was visualized by chemiluminescence (SuperSignal West Pico Chemiluminescent; Thermo Fisher Scientific) according to manufacturer's instructions. The intensity of the signals was quantified by VersaDoc Imaging system (Bio-Rad). The intensities of the immunostained bands were normalized with the total protein intensities measured by Coomassie brilliant blue $\mathrm{G}-250$ from the same blot.

Statistical analyses. Statistical analyses were carried out with the non-parametric Wilcoxon sign-rank test for paired samples for both 2-DE and western blot data. A p-value $<0.05$ was considered as statistically significant. Analyses were conducted with Stata/IC 12.2 for Windows (StataCorp LP, College Station, TX, USA).

\section{Results}

Proteomic studies. Using the 2-DE coupled with MS, a comparative proteomics analysis was performed between uterine leiomyoma and myometrium tissues. Correlation analysis of gel-pairs performed well, with average matching efficiency of about $75 \%$. An average of 2,200 spots was detected on gels for both types of proteomes. In this study 10 protein spots, belonging to cytoskeletal proteins with several biological functions, were found to be significantly dysregulated in leiomyoma samples compared to the myometrium. Eight spots were significantly upregulated ( $>1.5$-fold) and two were significantly downregulated ( $<0.6$ fold) (Fig. 1), and corresponded to 10 proteins identified by MALDI-TOF/TOF and LTQ-Orbitrap XL by searching the MS/MS data against the human section of the UniProt database (Table I). We found a significant increase in the leiomyoma of tubulin $\beta$ (TUBB), myosin regulatory light polypeptide 9 (MYL9), desmin (DES), four and a half LIM domains protein 1 (FHL1), keratin, type II cytoskeletal 1 (KRT1), keratin, type I cytoskeletal 9 (KRT9), LIM and SH3 domain protein 1 fragment (LASP1), actin $\alpha$ cardiac muscle 1 (ACTC1). Transgelin (TAGLN), prelamin A/C (LMNA) were found to be significantly downregulated in the leiomyoma compared to the myometrium.

Three of these proteins are involved in the promotion of cell migration (FHL1, LASP1 and MYL9), while TAGLN is involved in replicative senescence.

Validation of myosin regulatory light polypeptide 9. In this study we decided to validate myosin regulatory light polypeptide 9 (MYL9) because it is considered to be a cell migration marker, in several cancers and could play a key role in leiomyoma development. MYL9 expression in seven leiomyomas was compared to the expression in matched normal myometrial tissue samples by western blot analysis. MYL9 expression was significantly higher in the leiomyoma with respect to the myometrium, confirming results obtained from the 2-DE analysis. In Fig. 2 we report the quantitative analysis of MYL9 expression $(\mathrm{P}=0.031)$. We opted for a Coomassie staining protocol because, as described by Lv et al (13), and according to our results, the two housekeeping proteins, $\beta$-actin and tubulin, are upregulated in leiomyoma, and thus not adequate as controls. We do not have information on the expression of the other housekeeping proteins. 
Table I. Cytoskeletal protein expression levels measured by mass spectrometry in the leiomyoma and in the myometrium proteome.

\begin{tabular}{|c|c|c|c|c|c|c|}
\hline $\begin{array}{l}\text { Accession } \\
\text { no. }\end{array}$ & $\begin{array}{c}\text { Spot } \\
\text { no. }\end{array}$ & $\begin{array}{c}\text { Protein } \\
\text { description }\end{array}$ & $\begin{array}{l}\text { Gene } \\
\text { symbol }\end{array}$ & $\begin{array}{l}\text { Total } \\
\text { score }\end{array}$ & $\begin{array}{l}\text { Fold } \\
\text { change }^{\mathrm{a}}\end{array}$ & $\begin{array}{l}\text { Biological } \\
\text { function }\end{array}$ \\
\hline P68032 & 10 & Actin, $\alpha$ cardiac muscle 1 & ACTC1 & 248 & 3.6 & Cell motility \\
\hline Q13642 & 4 & Four and a half LIM domains protein 1 & FHL1 & 224 & 3.4 & Cell migration \\
\hline P04264 & 5 & Keratin, type II cytoskeletal 1 & KRT1 & 107 & 3.4 & Regulators of inflammation \\
\hline Q14847 & 9 & $\begin{array}{l}\text { LIM and SH3 domain protein } 1 \\
\text { fragment }\end{array}$ & LASP1 & 66 & 2 & Cell migration \\
\hline P24844 & 2 & $\begin{array}{l}\text { Myosin regulatory light } \\
\text { polypeptide } 9\end{array}$ & MYL9 & 100 & 1.8 & Cell migration \\
\hline P17661 & 3 & Desmin & DES & 190 & 1.6 & $\begin{array}{l}\text { Main component of } \\
\text { intermediate filaments }\end{array}$ \\
\hline P35527 & 8 & Keratin, type I cytoskeletal 9 & KRT9 & 214 & 1.6 & Keratin filament assembly \\
\hline P07437 & 1 & Tubulin $\beta$ chain & TUBB & 317 & 1.6 & Compnent of microtubules \\
\hline P02545 & 7 & Prelamin-A/C & LMNA & 120 & 0.5 & $\begin{array}{l}\text { Nuclear assembly/chromatin } \\
\text { organization }\end{array}$ \\
\hline Q01995 & 6 & Transgelin & TAGLN & 1,200 & 0.5 & Replicative senescence \\
\hline
\end{tabular}

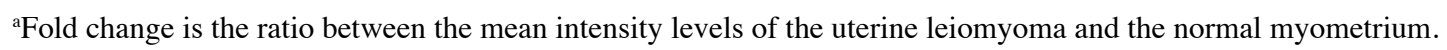

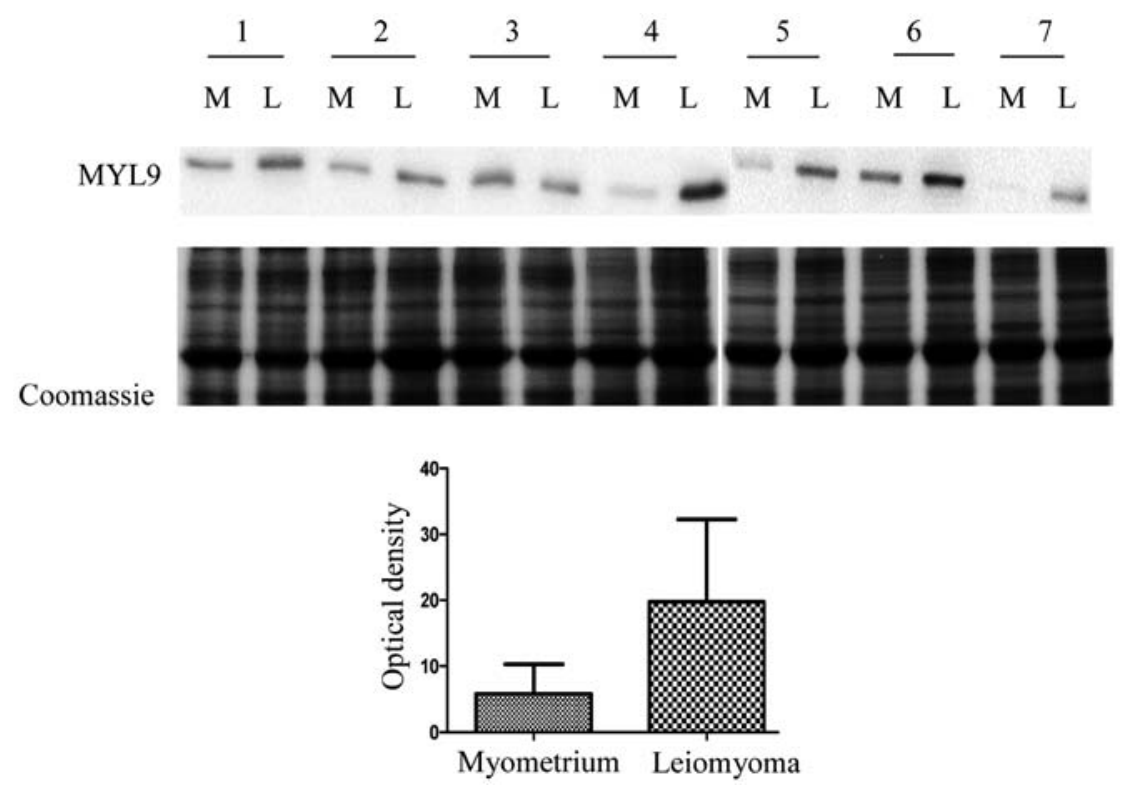

Figure 2. Western blot analysis of myosin regulatory light polypeptide 9 (MYL9) in paired myometrium (M) and leiomyoma (L). The intensity of immunostained bands was normalized against the total protein intensities measured from the same blot stained with Coomassie blue. The bar graph shows the relative expression (band density) of myosin regulatory light polypeptide 9 in the myometrium and the leiomyoma. Results are shown as a histogram $(\mathrm{P}<0.05)$ and each bar represents mean \pm SD.

Protein-protein interaction analysis. The dysregulated cytoskeletal related proteins identified in this study were loaded on STRING 9.0 to generate a prediction of protein-protein interaction networks. The strongest interactions were between: LASP1 and ACTC1 (combined score, 0.774), and KRT1 and KRT9 (combined score, 0.76) (Fig. 3A). We further analyzed the interaction between transforming protein RhoA (a key protein in the regulation of the signal transduction pathway involved in leiomyoma growth) and the proteins identified in our study (Fig. 3B).
The strongest evidence was the interaction between RHOA and MYL9 (combined score, 0.99). Another interesting result based on action prediction was the activation of MYL9 by RHOA (Fig. 3C).

\section{Discussion}

Several studies have highlighted the role of extracellular forces in the reorganization of the cytoskeleton in leiomyoma cells. Increases in mechanical stress induce an excessive ECM 
A

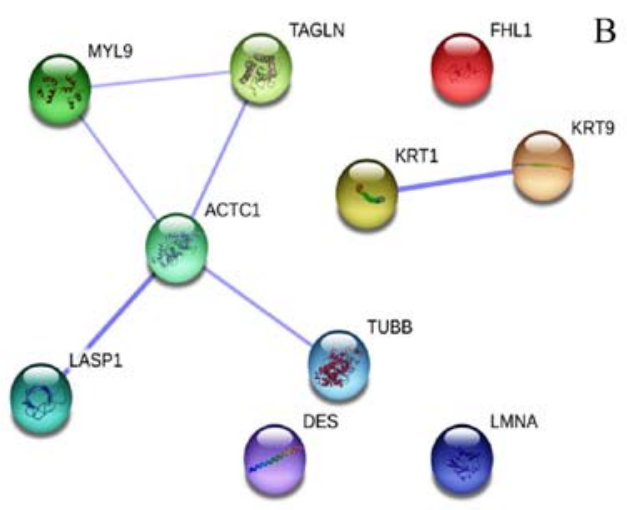

$\mathrm{C}$

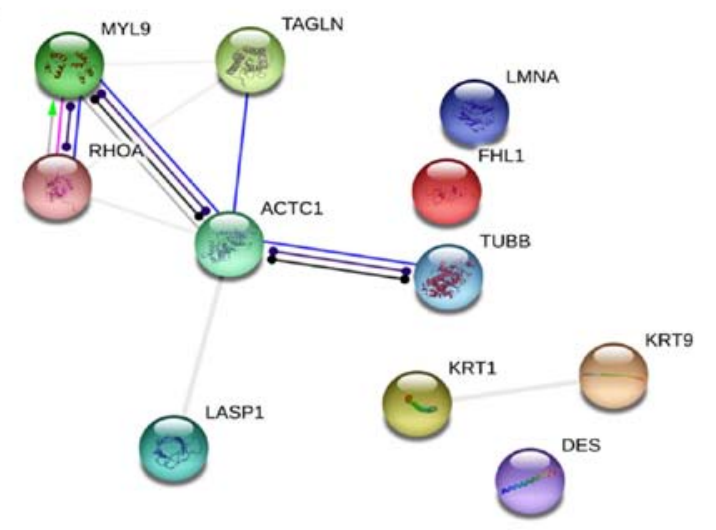

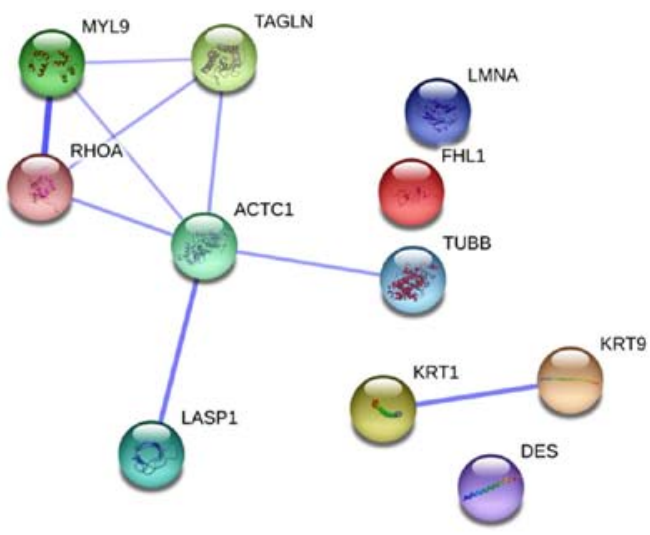

Legend

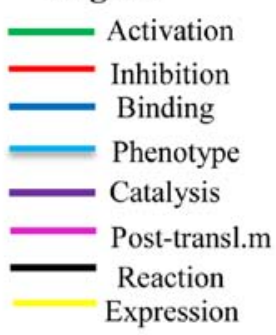

Figure 3. Prediction by STRING database. (A) Cytoskeletal protein interaction on confidence prediction. (B) Cytoskeletal and RHOA protein on confidence prediction. (C) Green arrow indicates activation of MYL9 from RHOA on activate prediction. Abbreviations are given in the text.

deposition and tumorigenesis promotion $(11,14)$. Cytoskeleton integrity plays an important role in cell cycle progression, death and differentiation, and abnormal cytoskeleton functions are often observed in leiomyoma cells (15).

To the best of our knowledge, this is the first proteomics study presenting data on the expression of several cytoskeletal proteins involved in cell migration.

Actins and tubulin are two major components of the cytoskeleton, with key roles in cell morphology (16). Disassembly of actin and microtubule dynamic instability may be associated to leiomyoma growth (17). A wide variety of anticancer drugs are able to bind to TUBB, interfering with the microtubule dynamics such as paclitaxel (18). The uterine leiomyoma consists mainly of smooth muscle cells with abundant expression of DES (19). The overexpression of desmin inside and outside of the cell certainly indicates its involvement in the mechanotransduction and in tumor development (10).

FHL1 regulates cytoskeleton-associated proteins (20) and its overexpression in myoblasts inhibits cell adhesion and promotes cell migration (21). Studies on FHL1 gene expression have shown that FHL1 knockdown induces inhibition of cell proliferation (21). These studies can help understanding of the possible involvement of FHL1 in leiomyoma cell proliferation and growth.

In the leiomyoma, integrin activation takes place by cell contractility and by ECM stiffening. Integrin leads to the activation of RHOA, which activates myosin light chain kinase.
Phosphorylation of MYL9 by myosin light chain kinase plays an important role in the regulation of smooth muscle cell contractile activity and is implicated in cytokinesis and cell locomotion $(4,22)$.

MYL9 is considered to be a cell migration marker in breast cancer and its expression is necessary for cytoskeletal dynamics and experimental metastasis $(23,24)$. For the first time, in this study we report the validation of MYL9 expression by western blot, confirming the 2-DE result. The STRING interaction network shows the activation of MYL9 by RHOA, and this result is corroborated by the literature.

Keratins constitute the intermediate filament of the cytoskeleton in the epithelia and KRT1 is one of the major constituents of the cytoskeleton of keratinocytes (25). KRT1 plays an essential role in preserving the integrity of the cell from mechanical stress, and in the protection of the cell from inflammation (25). KRT9 is another constituent of the intermediate filament cytoskeleton identified in our study revealing overexpression of this protein in the leiomyoma with respect to the normal myometrium.

LASP1, an actin-binding protein, plays a role in the organization of the cytoskeleton, is involved in a signaling pathway and in the regulation of cell migration and proliferation (26). Our STRING network data confirm the interaction between LASP1 and actin. Specific phosphorylation of LASP1 at serine/threonine and tyrosine regulates the function and the localization of the protein. LASP1 is overexpressed in breast 
and ovarian cancer, promoting cell migration and proliferation (27). This evidence supports our results regarding the overexpression of LAPS1 in the leiomyoma compared to the normal myometrium.

ACT1 belongs to the actin isoforms $\alpha$, which are found in muscle tissues and are a major constituent of the contractile apparatus (28). This protein is recruited at early stages of cell adhesion (29). Previously published studies have found an interaction between $\alpha$-actinin and actin, and have suggested a possible role of $\alpha$-actinin in adhesion maturation (29). In the leiomyoma, the overexpression of ACTC1 can lead to the dysregulation of the cytoskeleton, inducing proliferation and growth $(4,22)$.

Finally, we found that LAMN A/C and TAGLN are downregulated in the leiomyoma proteome. Our results are in line with our previous study on interstitial fluid (10).

Lamins constitute a class of intermediate filament with structural and functional roles, involved in dynamic chromatin organization and gene transcription (30). Increasing of mechanical forces in ECM may induce a downregulation on lamin, inducing chromatin remodeling and dysregulation of gene expression involved in cell proliferation and adhesion (31).

Transgelin is an abundant protein of smooth muscle cells involved in the stabilization of actin filaments and is directly and indirectly involved in many cancer-related processes such as proliferation, migration, differentiation and apoptosis (32). Evidence shows how transgelin acts as a tumor suppressor (33) and its downregulation in the leiomyoma promotes cell proliferation, and is consequently associated with the growth of the fibroid tumor (34).

In conclusion, the 2-DE approach remains the technique of choice for comparative proteomic.

Our data demonstrate significant alterations in the expression of cytoskeletal proteins in the leiomyoma tissue compared to the normal myometrium. These proteins are involved in cell migration, tumor growth and cell proliferation. All the dysregulated cytoskeletal proteins may be directly involved in signaling pathway, contributing to the development of the leiomyoma. A better understanding of the involvement of cytoskeletal proteins in leiomyoma growth may help identify new therapeutic targets for the development of new pharmacological approaches.

\section{References}

1. Rein MS: Advances in uterine leiomyoma research: The progesterone hypothesis. Environ Health Perspect 108 (Suppl 5): 791-793, 2000.

2. Catherino WH and Malik M: Uterine leiomyomas express a molecular pattern that lowers retinoic acid exposure. Fertil Steril 87: 1388-1398, 2007.

3. Arslan AA, Gold LI, Mittal K, Suen TC, Belitskaya-Levy I, Tang MS and Toniolo P: Gene expression studies provide clues to the pathogenesis of uterine leiomyoma: New evidence and a systematic review. Hum Reprod 20: 852-863, 2005.

4. Leppert PC, Jayes FL and Segars JH: The extracellular matrix contributes to mechanotransduction in uterine fibroids. Obstet Gynecol Int 2014: 783289, 2014.

5. Davis BJ, Risinger JI, Chandramouli GV, Bushel PR, Baird DD and Peddada SD: Gene expression in uterine leiomyoma from tumors likely to be growing (from black women over 35) and tumors likely to be non-growing (from white women over 35 ). PLoS One 8: e63909, 2013.
6. Wang Y, Feng G, Wang J, Zhou Y, Liu Y, Shi Y, Zhu Y, Lin W, $\mathrm{Xu}$ Y and Li Z: Differential effects of tumor necrosis factor- $\alpha$ on matrix metalloproteinase-2 expression in human myometrial and uterine leiomyoma smooth muscle cells. Hum Reprod 30: 61-70, 2015

7. Elosegui-Artola A, Bazellières E, Allen MD, Andreu I, Oria R, Sunyer R, Gomm JJ, Marshall JF, Jones JL, Trepat X, et al: Rigidity sensing and adaptation through regulation of integrin types. Nat Mater 13: 631-637, 2014.

8. Vincent $\mathrm{S}$ and Settleman J: The PRK2 kinase is a potential effector target of both Rho and Rac GTPases and regulates actin cytoskeletal organization. Mol Cell Biol 17: 2247-2256, 1997.

9. Luo XG, Zhang CL, Zhao WW, Liu ZP, Liu L, Mu A, Guo S, Wang N, Zhou $\mathrm{H}$ and Zhang TC: Histone methyltransferase SMYD3 promotes MRTF-A-mediated transactivation of MYL9 and migration of MCF-7 breast cancer cells. Cancer Lett 344: 129-137, 2014.

10. Ura B, Scrimin F, Zanconati F, Arrigoni G, Monasta L, Romano A, Banco R, Zweyer M, Milani D and Ricci G: Two-dimensional gel electrophoresis analysis of the leiomyoma interstitial fluid reveals altered protein expression with a possible involvement in pathogenesis. Oncol Rep 33: 2219-2226, 2015.

11. Norian JM, Owen CM, Taboas J, Korecki C, Tuan R, Malik M, Catherino WH and Segars JH: Characterization of tissue biomechanics and mechanical signaling in uterine leiomyoma. Matrix Biol 31: 57-65, 2012.

12. Mischiati C, Ura B, Roncoroni L, Elli L, Cervellati C, Squerzanti M, Conte D, Doneda L, Polverino de Laureto P, de Franceschi G, et al: Changes in protein expression in two cholangiocarcinoma cell lines undergoing formation of multicellular tumor spheroids in vitro. PLoS One 10: e0118906, 2015.

13. Lv J, Zhu X, Dong K, Lin Y, Hu Y and Zhu C: Reduced expression of 14-3-3 $\gamma$ in uterine leiomyoma as identified by proteomics. Fertil Steril 90: 1892-1898, 2008.

14. Mouw JK, Yui Y, Damiano L, Bainer RO, Lakins JN, Acerbi I, Ou G, Wijekoon AC, Levental KR, Gilbert PM, et al: Tissue mechanics modulate microRNA-dependent PTEN expression to regulate malignant progression. Nat Med 20: 360-367, 2014.

15. Butcher DT, Alliston T and Weaver VM: A tense situation: Forcing tumour progression. Nat Rev Cancer 9: 108-122, 2009.

16. Cooper JA: The role of actin polymerization in cell motility. Annu Rev Physiol 53: 585-605, 1991.

17. Salama SA, Kamel MW, Botting S, Salih SM, Borahay MA, Hamed AA, Kilic GS, Saeed M, Williams MY and Diaz-Arrastia CR: Catechol-o-methyltransferase expression and 2-methoxyestradiol affect microtubule dynamics and modify steroid receptor signaling in leiomyoma cells. PLoS One 4: e7356, 2009.

18. Ganguly A, Yang H and Cabral F: Class III $\beta$-tubulin counteracts the ability of paclitaxel to inhibit cell migration. Oncotarget 2: 368-377, 2011.

19. Roeder HA, Cramer SF and Leppert PC: A look at uterine wound healing through a histopathological study of uterine scars. Reprod Sci 19: 463-473, 2012.

20. Brown S, McGrath MJ, Ooms LM, Gurung R, Maimone MM and Mitchell CA: Characterization of two isoforms of the skeletal muscle LIM protein 1, SLIM1. Localization of SLIM1 at focal adhesions and the isoform slimmer in the nucleus of myoblasts and cytoplasm of myotubes suggests distinct roles in the cytoskeleton and in nuclear-cytoplasmic communication. J Biol Chem 274: 27083-27091, 1999.

21. Robinson PA, Brown S, McGrath MJ, Coghill ID, Gurung R and Mitchell CA: Skeletal muscle LIM protein 1 regulates integrin-mediated myoblast adhesion, spreading, and migration. Am J Physiol Cell Physiol 284: C681-C695, 2003.

22. Huveneers S and Danen EH: Adhesion signaling - crosstalk between integrins, Src and Rho. J Cell Sci 122: 1059-1069, 2009.

23. Liao XH, Wang N, Liu LY, Zheng L, Xing WJ, Zhao DW, Sun XG, Hu P, Dong J and Zhang TC: MRTF-A and STAT3 synergistically promote breast cancer cell migration. Cell Signal 26: 2370-2380, 2014.

24. Medjkane S, Perez-Sanchez C, Gaggioli C, Sahai E and Treisman R: Myocardin-related transcription factors and SRF are required for cytoskeletal dynamics and experimental metastasis. Nat Cell Biol 11: 257-268, 2009.

25. Roth W, Kumar V, Beer HD, Richter M, Wohlenberg C, Reuter U, Thiering S, Staratschek-Jox A, Hofmann A, Kreusch F, et al: Keratin 1 maintains skin integrity and participates in an inflammatory network in skin through interleukin-18. J Cell Sci 125: 5269-5279, 2012. 
26. Dandapani SV, Sugimoto H, Matthews BD, Kolb RJ, Sinha S, Gerszten RE, Zhou J, Ingber DE, Kalluri R and Pollak MR: $\alpha$-actinin- 4 is required for normal podocyte adhesion. $\mathrm{J}$ Biol Chem 282: 467-477, 2007.

27. Grunewald TG and Butt E: The LIM and SH3 domain protein family: Structural proteins or signal transducers or both? Mol Cancer 7: 31, 2008.

28. Matsson H, Eason J, Bookwalter CS, Klar J, Gustavsson P, Sunnegårdh J, Enell H, Jonzon A, Vikkula M, Gutierrez I, et al: $\alpha$-cardiac actin mutations produce atrial septal defects. Hum Mol Genet 17: 256-265, 2008

29. Choi CK, Vicente-Manzanares M, Zareno J, Whitmore LA, Mogilner A and Horwitz AR: Actin and $\alpha$-actinin orchestrate the assembly and maturation of nascent adhesions in a myosin II motor-independent manner. Nat Cell Biol 10: 1039-1050, 2008.

30. Dechat T, Adam SA, Taimen P, Shimi T and Goldman RD Nuclear lamins. Cold Spring Harb Perspect Biol 2: a000547, 2010.
31. Iyer KV, Pulford S, Mogilner A and Shivashankar GV: Mechanical activation of cells induces chromatin remodeling preceding MKL nuclear transport. Biophys J 103: 1416-1428, 2012.

32. Dvorakova M, Nenutil R and Bouchal P: Transgelins, cytoskeletal proteins implicated in different aspects of cancer development. Expert Rev Proteomics 11: 149-165, 2014.

33. Yang Z, Chang YJ, Miyamoto H, Ni J, Niu Y, Chen Z, Chen YL, Yao JL, di Sant'Agnese PA and Chang C: Transgelin functions as a suppressor via inhibition of ARA54-enhanced androgen receptor transactivation and prostate cancer cell growth. Mol Endocrinol 21: 343-358, 2007.

34. Li Q, Shi R, Wang Y and Niu X: TAGLN suppresses proliferation and invasion, and induces apoptosis of colorectal carcinoma cells. Tumour Biol 34: 505-513, 2013. 